\title{
Editorial
}

\section{Memórias and the Journal Citation Reports}

We, as many other journal editors, have been looking at the recent release of the 2014 Journal Citation Reports by Thompson-Reuters. The results for the Memórias do Instituto Oswaldo Cruz have again been gratifying; our twoyear impact factor has increased to 1.592 while the Memórias is one of the few Latin American journals with a fiveyear impact factor above 2.0. The other indices released by Thomson-Reuters have provided even more impressive news for the Memórias. The journal continues not only to be the most highly cited scientific periodical (with 5,899 citations in 2014), but also has the highest Eigenfactor score (0.086) of any Latin American journal. The Eigenfactor is like the impact factor, but gives more weight to citations from highly cited journals and less weight to citations from less cited journals, and self-citations are also omitted from the calculation.

However when we compare the Memórias to international journals worldwide the results are not so impressive. Here we have the same struggle as many other journals published in developing countries. That is, how can we attract the best manuscripts from our region, which tend to be sent by their authors to highly cited international journals? Even so the Memórias, in the tropical medicine category is classified within the top ten journals, using ranking by any of the indices mentioned above, while among parasitology journals the Memórias is ranked in the third quartile by impact factor. Tropical medicine and parasitology are the only two categories were Thomson-Reuters classifies the Memórias.

Other data from the Journal Citation Reports show the changes in journal publishing. One is the growth of megajournals. There are now over 100 journals that publish 1,000 articles per year. PLoS One, that published more than 30,000 articles last year, heads the list. While many are traditional journals, such as Proceedings of the National Academy of Sciences and the Journal of Biological Chemistry, there are several newer open access (OA) journals as for instance the Scientific Reports from the Nature Publishing Group, which published nearly 4,000 articles with an impact factor of over 5.0.

Another trend has been the rise of OA journals. In several categories, such as public health, tropical medicine and parasitology, the ranking of journals by impact factor is headed by OA journals. New OA journals such as eLife, which is sponsored by major research funders, have received their first impact factors (an impressive score of nearly 10) and flagship journals published by the major OA publishers have seen increases in their impact.

Brazil is fortunate to have most of its scientific journals published as OA. This is principally due to the SciELO project, which provides an OA platform for selected Brazilian journals, including the Memórias.

Many of the Brazilian journals, including the Memórias, are published under a special model of OA where no fees are charged to authors and readers sometimes referred to as Platinum Open Acess. These journals are funded by a variety of public resources (including institutional and research agency funding). This together with the pro bono work of their editors and reviewers allows for a form of open public access: authors can contribute to the scientific literature and any member of the public can access this literature without any financial barriers. The scientific publication process is thus funded by the Brazilian taxpayers as a logical complement to their investment in scientific research.

With this distinctive form of OA together with improvements in the quality of its contents and better dissemination of the journal we hope that the Memórias will continue to increase its impact in the coming years. 